\title{
Médiévales
}

Langues, Textes, Histoire

75 | automne 2018

Traductions du Moyen Âge

\section{Le choix des mots. Traduire les textes érotiques du Moyen Âge}

A choice of words? Translating the erotic texts of the Middle Ages

\section{Corinne Pierreville}

\section{(2) OpenEdition}

1 Journals

\section{Édition électronique}

URL : https://journals.openedition.org/medievales/9411

DOI : 10.4000/medievales. 9411

ISSN : 1777-5892

Éditeur

Presses universitaires de Vincennes

\section{Édition imprimée}

Date de publication : 15 octobre 2018

Pagination : 151-166

ISBN : 978-2-84292-861-2

ISSN : 0751-2708

Référence électronique

Corinne Pierreville, «Le choix des mots. Traduire les textes érotiques du Moyen Âge », Médiévales [En ligne], 75 | automne 2018, mis en ligne le 15 octobre 2019, consulté le 22 avril 2022. URL : http:// journals.openedition.org/medievales/9411; DOI : https://doi.org/10.4000/medievales.9411 


\section{Corinne Pierreville}

\section{Le choix des mots Traduire les textes érotiques du Moyen Âge}

La traduction est, comme chacun sait, un exercice condamnant le traducteur à une forme de frustration : il est impossible de restituer parfaitement toutes les nuances d'un texte, les connotations d'un mot, le rythme, les sonorités, les suggestions phoniques et sémantiques, le style d'un auteur. En bref, on ne peut dire exactement dans une langue ce qui a été dit dans une autre langue ou dans la même langue neuf siècles plus tôt. Traduire, c'est au pire trahir, comme le suggèrent le traducteur, traditeur de Joachim du Bellay ou le traduttore, traditore italien assimilant le traducteur au traître ${ }^{1}$. C'est au mieux «dire presque la même chose » si l'on possède le talent d'un Umberto Eco ${ }^{2}$. Pendant longtemps d'ailleurs, traduire les œuvres littéraires médiévales était considéré comme accessoire, tout lecteur respectable étant implicitement tenu de comprendre l'ancien français. On pourrait étendre ce principe à l'ensemble des langues mortes ou vivantes : dans un monde idéal, nous devrions tous être polyglottes et goûter Shakespeare, Cervantès, Ovide, Homère et Chrétien de Troyes dans le texte. Mais dans le monde tel qu'il est, il faut traduire, et les difficultés de cette entreprise nous paraissent s'accroître quand le texte source relève d'une littérature s'attachant à l'évocation du désir et du plaisir sexuel.

1. Voir J. Du Bellay, La Défense et illustration de la langue française, Paris, 1905, chap. VI, p. 76 : " Mais que diray-je d'aucuns, vrayement mieux dignes d'estre appelez traditeurs, que traducteurs ? Veu qu'ils trahissent ceux qu'ils entreprennent exposer, les frustrans de leur gloire, et par mesme moyen seduisent les lecteurs ignorans, leur monstrant le blanc pour le noir. » L'origine de l'expression italienne est controversée. Voir B. CoHEN, « Traduttore... traditeur ? Les mystères d'une formule rabâchée » (2008), [en ligne] http:// archive.is/OoEyM [consulté le 19 septembre 2018].

2. Voir U. Eco, Dire presque la même chose. Expériences de traduction, trad. de l'italien par M. BouzAHER, Paris, 2006. 
Lors de la constitution d'une anthologie de la littérature érotique médiévale ${ }^{3}$, la nécessité de ces traductions s'est imposée afin d'éviter de surcharger les textes de notes sémantiques, tant la langue du Moyen Âge est devenue étrangère au lecteur contemporain ${ }^{4}$. Deux exemples pourraient suffire à le montrer. En ancien français, le verbe baiser ne possède pas le sens moderne populaire impliquant une possession charnelle, mais signifie seulement " embrasser, donner un baiser ». A contrario, le verbe foutre renvoyait précisément à l'activité sexuelle et au coït, alors qu'il est si souvent employé de nos jours comme synonyme de faire dans la locution vulgaire «n'en avoir rien à foutre ». Traduire est donc nécessaire pour éviter au lecteur des erreurs de compréhension et lui donner les clés du sens. Le choix des termes utilisés peut cependant se révéler lourd de conséquences. Il ne s'agit pas seulement de traduire les textes érotiques du Moyen Âge, mais de sélectionner avec soin les termes pour le faire. On pourrait ainsi s'amuser à imaginer au moins trois traductions possibles des conseils fournis en catalan par l'auteur du Speculum al foderi à son lecteur confronté à une femme peu encline au coït :

Ti estranga li lo cony bé, e la torça, a la pecich entro que crit, es sacuda, es bé plagua. E ab aço, la encendra que haura desig de foder [...]. Item [...] torça la e pessich la ab la ma en lo cony, e vendra li lo talent. Puys posa li la verga en la vora del cony entro que sia encesa e scalfada.

Malaxe-lui bien le con, frotte-le et masse-le jusqu'au moment où elle poussera des cris et des gémissements et où elle tremblera. Elle sera alors excitée et elle aura envie de baiser [...]. Frotte aussi son con avec ta main, masse-le et elle aura envie de baiser. Puis pose ta bite à l'entrée de son con jusqu'à ce qu'elle devienne chaude et qu'elle soit excitée. (1)

Malaxe son sexe, frotte-le et masse-le jusqu'à la faire gémir, trembler et crier. Elle sera alors encline à désirer le coït $[\ldots]$. De même [...] frotte et masse son sexe de ta main et l'excitation naitra en elle. Puis place ta verge à l'entrée de son sexe jusqu'à ce qu'elle s'échauffe et s'excite. (2)

Malaxe son sexe, frotte-le et masse-le jusqu'à la faire gémir, trembler et crier. Elle désirera alors être pénétrée [...]. De même [...] frotte et masse son sexe de ta main et elle sera prise de désir. Puis place ta verge à l'entrée de son sexe jusqu'à ce que tout son corps s'enflamme. (3)

3. C. Pierreville, Anthologie de la littérature érotique du Moyen Âge, à paraitre aux éditions Champion. Sauf mention contraire, les textes sont cités d'après ce recueil.

4. Il est néanmoins un texte où nous avons dû nous résoudre à insérer des notes de bas de page pour éclairer le sens de la traduction, la ballade Marion, entendez-moi d'Eustache Deschamps, en raison du vocabulaire musical spécifique utilisé par le poète. 
Traduire cony par « con » ou par « sexe », verga par «bite » ou « verge », desig de foder par « envie de baiser », « désirer le coït » ou « désirer être pénétrée », enceser et scalfader par « devenir chaude et être excitée », « s'échauffer et s'exciter » ou «brûler de désir », modifie radicalement la tonalité de l'extrait qui varie du synopsis pornographique (1), au livre érotique (3) en passant par le traité de sexologie (2). C'est évidemment la coloration d'ensemble du texte source qui guide les choix du traducteur. Comme le Speculum al foderi est un traité de médecine de la fin du XIV ou du début du $\mathrm{XV}^{\mathrm{e}}$ siècle, il n'est pas question que sa traduction sombre dans la pornographie. Mais dans la mesure où il s'agit du tout premier ouvrage occidental à proposer un art des positions sexuelles jamais détaillées avant lui, le traducteur est autorisé à s'éloigner d'un style purement médical pour laisser résonner l'érotisme affleurant dans le texte catalan ${ }^{5}$.

La première question à résoudre tient ainsi au choix des termes désignant les parties intimes du corps. Leur charge obscène a en effet varié avec le temps, comme l'atteste le mot cul qui appartient de nos jours au langage grossier, alors qu'il ne possédait pas de synonyme neutre dans la langue médiévale, qui ignorait l'emploi des substantifs « postérieur », « arrière-train » ou « fondement ». Le terme « croupe » existait, mais il était aussi familier qu'actuellement. La présence du substantif cul dans des livres de médecine ou des textes sérieux du Moyen Âge prouve au reste qu'il relevait du langage commun ${ }^{6}$. Si le traducteur décide de maintenir ce substantif, il doit être certain que le texte use à escient d'un niveau de langue trivial. Les érudits du passé avaient trouvé une méthode radicale pour résoudre ce type de problème. Ils remplaçaient les passages jugés licencieux par des points de suspension, comme Alfred Jeanroy face aux strophes les plus lestes de Guillaume IX d'Aquitaine ${ }^{7}$. Ils supprimaient aussi les termes incriminés ou leur substituaient des équivalents qui ne manquent pas de faire sourire parfois a posteriori. Dans son édition des fabliaux ${ }^{8}$, Joseph Bédier transforme le Pet au vilain en L'Âme au vilain, il abrège Bérangier au long cul, remplace les cons par des muets dans Le chevalier qui fist parler les cons et transforme élégamment Celle qui se fit foutre sur la fosse de son mari en La femme au tombeau. S'il est évident qu'on ne peut plus de nos jours dénaturer ainsi les

5. C'est la voie moyenne trouvée dans la traduction que nous proposons dans notre anthologie pour ce passage : « Malaxe son sexe, frotte-le et masse-le jusqu'à ce qu'elle commence à gémir, trembler et crier. Ainsi sera-t-elle incitée à désirer la relation sexuelle [...] De même [...], pétris et masse son sexe avec ta main et le désir naîtra en elle. Puis place ta verge à l'entrée de son sexe jusqu'à ce qu'elle brûle de désir. »

6. Voir P. MÉnARD, Les Fabliaux, contes à rire du Moyen Âge, Paris, 1983, p. 151 ; ID., Le Rire et le sourire dans le roman courtois en France au Moyen Âge (1150-1250), Genève, 1969, p. 692.

7. Voir A. Jeanroy, Les Chansons de Guillaume IX duc d'Aquitaine, Paris, 1913, p. 8-13.

8. J. BÉDIER, Les Fabliaux, études de littérature populaire et d'histoire littéraire du Moyen Âge, Paris, 1893. 
sources littéraires en gommant les mots qui choquent notre pudeur, la question de leur traduction reste entière.

Un examen attentif des vingt-six textes composant l'anthologie révèle que les termes con, cul, couille, vit et foutre n'apparaissent guère. La raison en est simple : contrairement à ce que pensait Sainte-Beuve, persuadé qu'on pouvait tout nommer au Moyen Âge « avec une sorte d'effronterie naïve ${ }^{9}$ », la littérature de style soutenu bannissait l'emploi des mots référant aux parties sexuelles et aux besoins primaires du corps. Dans le Roman de la Rose de Guillaume de Loris, le dieu Amour interdit ainsi à l'amant de les prononcer ${ }^{10}$ :

Aprés gardes que tu ne dies ces orz moz ne cez ribaudies :

ja por nomer vilainne chose ne doit ta bouche estre declouse.

Je ne tien pas a cortois home

qui orde chose et laide nome.

L'auteur insiste sur l'idée d'ordure et d'ignominie attachée à ces termes que la bouche du parfait amant ne devra jamais laisser échapper. Seul un traité médical comme le Speculum al foderi est en droit d'user de certains d'entre eux en l'absence d'équivalent neutre ou non marqué. Dans les autres textes de l'anthologie, leur présence signale toujours une forme de transgression à l'égard de la bienséance et de la pudeur ${ }^{11}$. Ils rompent avec les usages de la littérature courtoise ; ils suscitent chez l'auditoire une réaction de surprise, le choquent ou l'amusent. Le traducteur ne peut les conserver que si leurs connotations triviales sont immédiatement perceptibles en français moderne.

Le substantif con pâtit ainsi à nos yeux de l'ambiguïté générée de nos jours par son emploi figuré dans la langue vulgaire pour désigner une personne stupide. Quand nos contemporains évoquent de « sales cons », ils ne pensent pas un instant au niveau de propreté des organes génitaux externes de la femme. Mais dans la mesure où ce substantif est encore employé dans le sens sexuel qui était le sien en ancien français, il a été conservé dans tous les textes où sa

9. SaInte-Beuve, Tableau historique et critique de la poésie française et du théâtre français au XVI siècle, Paris, 1838, p. 175.

10. Guillaume De Lorris, Le Roman de la Rose, éd. F. LeCoy, Paris, 1983, v. 2097-2102.

11. Nous renvoyons uniquement à la première mention du terme dans les extraits cités. Coille, couille : Confession de Margot, v. 86 ; Demoiselle qui ne pouvait entendre parler de foutre, v. 23 ; Sot chevalier, v. 92 ; Trubert, v. 2404. Coillon : Tenzon du seigneur Montant et de la dame, v. 13 ; Demoiselle qui ne pouvait entendre parler de foutre, v. 178. Con : Tenzon du seigneur Montant et de la dame, v. 7 ; Demoiselle qui ne pouvait entendre parler de foutre, v. 204 ; Sot chevalier, v. 33 ; Trubert, v. 2506. Cul : Tenzon du seigneur Montant et de la dame, v. 6 ; Sot chevalier, v. 276. Culada : Tenzon du seigneur Montant et de la dame, v. 26. Culeter: Sot chevalier, v. 78. Fotre, foutre, foder : Tenzon du seigneur Montant et de la dame, v. 3 ; Demoiselle qui ne pouvait entendre parler de foutre, v. 6 ; Demoiselle qui songeait, v. 17 ; Farai un vers pos mi sonelh, v. 79 ; Richeut, v. 934 ; Saineresse, v. 44 ; Sot chevalier, v. 87. 
charge obscène était indéniable, comme lorsque Trubert, travesti en femme, se trouve dans le lit du roi Golias qui veut à toute force le posséder. Profitant de l'obscurité de la pièce, le héros se crée un substitut de sexe féminin en plaçant entre ses jambes une bourse de cuir dont il resserre brutalement les cordons autour de la verge de Golias, qui finit par s'évanouir de douleur :

Quant il revint de pasmoison : «Par foi, ainz mes ne vi tel con, fait li rois, ne sai dont ce vient !» Et Trubert qui molt bien le tient : «Sire, c'est un con de bïais ; si fet con ne verroiz jamais. » (v. 2849-54)
Quand il revint à lui :

« Ma foi, je n'ai jamais vu un tel con, fait le roi,j'ignore ce qui se passe ! » Et Trubert qui le tient fort bien : « Sire, c'est un con de biais ; vous n'en verrez jamais de tel. »

En conservant le terme con, la traduction maintient le comique verbal lié à l'emploi d'un mot vulgaire par un personnage aristocratique, le roi Golias, ou se donnant pour tel, Trubert ayant endossé l'identité de la fille du duc de Bourgogne. Selon un processus de démythification des pugnatores, le roi Golias est placé dans une situation inconvenante qui le dépare de son aura prestigieuse, conformément aux mécanismes du burlesque. La traduction voile cependant la diérèse que l'ancien français était tenue de prononcer sur le substantif biais pour que l'octosyllabe soit juste, privant le lecteur du jeu sémantique institué par ce préfixe $b i$-, désignant dès le latin ce qui est composé de deux ensembles identiques. Dans ce contexte réunissant deux hommes sur une même couche dans un simulacre de pénétration, fautil vraiment croire que la diérèse médiévale sur $b i$ - était innocente ?

Le substantif con a donc été conservé dans la traduction de tous les textes de l'anthologie où il instaure une distance souriante ou satirique à l'égard de la littérature officielle. Son équivalent catalan cony employé dans le Speculum al foderi ne pouvait en revanche être simplement décalqué, car il n'était pas question de surajouter des connotations familières ou populaires à cet ouvrage médical. Son emploi constant dans ce traité pour désigner le sexe de la femme prouve que le terme n'était pas marqué en lui-même et que l'ancienne langue n'en possédait pas d'autre afin de nommer l'organe sexuel féminin. Dans ce cas, une traduction neutre par « sexe » a été préférée à des terminologies plus anatomiques comme $«$ vagin $»$ ou $«$ vulve $»^{12}$.

Un cas particulier est posé par les noms propres inventés par les auteurs à partir de la personnification des organes génitaux de l'homme ou de la femme, Connebert dans le Sot chevalier et Couillebaude dans Trubert. Gautier Le Leu utilise le premier pour désigner le sexe féminin dans une expression emplie d'une déférence plaisante, dant Connebert. Ce patronyme ajoute au substantif con le suffixe -bert issu du germanique -berht qui signifiait

12. C'est également le choix effectué par P. Gifreu dans sa traduction : Miroir du foutre. Le Kamasutra catalan, Perpignan, 1995. 
« illustre », « brillant », et se trouvait fréquemment dans les noms du Moyen Âge comme Gombert, Guibert, Hildebert, Norbert, Robert, Bertrand, etc. Dès lors que certains de ces prénoms sont encore en usage de nos jours, il nous a paru possible de conserver Connebert tel quel dans la traduction, même si le lecteur moderne ne reconnait sans doute pas d'emblée l'allusion sexuelle ${ }^{13}$. Il fallait en revanche traduire le prénom Couillebaude dont s'affuble Trubert quand il est recueilli à la cour du duc de Bourgogne sous un travestissement féminin, car l'adjectif baud n'est plus compris actuellement. Or il était polysémique en ancien français et pouvait signifier « joyeux », «plein d'allégresse et d'ardeur », « fier » ou « hardi ${ }^{14}$, qualificatifs qui semblent tous appropriés pour caractériser les parties intimes du héros dont la sexualité n'inspire pas la mélancolie et qui est toujours prêt à forniquer. C'est finalement la traduction par « Couillehardie » qui a été retenue, car il nous a semblé que Douin de Lavesne avait formé ce patronyme à partir d'autres noms féminins connus de son auditoire. Couillebaude fait songer par ses sonorités finales à Aude, prénom d'ailleurs utilisé dans le roman pour désigner la suivante de la duchesse. Il existe de surcroît d'autres prénoms médiévaux dans lesquels on peut croire entendre un terme trivial, Constance, qui est précisément le nom de la gouvernante, ou Cunégonde, tiré du germanique kühn, « hardi » et de gund, «le combat ». La traduction par «Couillehardie » privilégie la même idée de vaillance, de témérité, et ses sonorités finales en -ie sont courantes dans les prénoms féminins - la gouvernante proposera d'ailleurs de rebaptiser Couillebaude « Fleurie » quand les demoiselles ne sont pas seules.

S'il est ainsi possible de transposer les substantifs con, couille et cul dans la majorité des textes de l'anthologie où ils apparaissent, le problème se complexifie quand on en arrive au substantif vit. La solution de facilité aurait consisté à le maintenir dans les traductions, comme le font la plupart des traducteurs ${ }^{15}$, mais à la différence de con, le terme vit est rare et vieilli. Son sens n'est donc plus immédiatement perceptible et sa présence dans la traduction risquait de générer des confusions ou des obscurités absentes du texte source. Jean-Luc Leclanche l'analyse avec justesse ${ }^{16}$ :

13. Il était néanmoins possible de créer un équivalent dont les connotations obscènes auraient été immédiatement perceptibles au lecteur contemporain en traduisant Connebert par Vulvebert, dont les sonorités peuvent évoquer le prénom masculin Fulbert.

14. F. Godefroy, Dictionnaire de l'ancienne langue française, Paris, 1881-1902, vol. I, p. 561, [en ligne] : http://micmap.org/dicfro/search/dictionnaire-godefroy/bald [consulté le 19 septembre 2018].

15. Voir P. BEC éd. et trad., Burlesque et obscénité chez les troubadours. Pour une approche du contre-texte médiéval, Paris, 1984, p. 163 ; R. BrusEgan, Fabliaux, Paris, 1994 ; L. Rossi, Fabliaux érotiques, textes de jongleurs des XII et XIII siècles, Paris, 1993 ; P. UhL, « La tenso entre Montan et une Dame (P.-C. 306, 2) : petit dialogue obscène entre amics fins », Expressions, 31 (2008), p. 67-85 (p. 72-73).

16. J.-L. LeClanche, Le Chevalier paillard. Quinze fabliaux libertins de chevalerie, Paris, 2008, p. 174. 
Le terme médiéval vit, contrairement au terme con qui désigne encore le sexe féminin, est aujourd'hui archaïque, son emploi est limité à la tradition des chansons de carabins ; le conserver dans la traduction aurait été un faux-fuyant.

Traduire en conservant le substantif vit, c'est en fait voiler le sens, c'est en atténuer la portée transgressive, c'est préserver le lecteur du choc de la confrontation crue avec les réalités corporelles et sexuelles que souhaitaient instaurer les auteurs. Mais alors, comment traduire et quel terme choisir ? Fallait-il opter pour le registre neutre de « verge », « sexe » ou « membre viril »? Préférer les sonorités latines de «phallus », les connotations anatomiques de « pénis », l'argot de «bite» ou de « queue»? Devait-on retenir au demeurant une seule et même traduction, quel que soit le texte source, par souci de cohérence ? La tonalité générale des extraits a dicté nos choix. Dans la tenzon de Montant, le recours assumé à l'obscénité justifie une traduction d'un niveau de langue vulgaire :

Et eu vas vos, dompn', ab braga bassada,

Ab mazer viet de nuill aiz'en despan,

E fotrai vos de tal arandonada Que los linzols storzeriez lendeman E pos diretz c'ops i es la bugada, Ni mais no'm leu ni mei coillon gran,

Se tan no'us fet que vos zaires pasmada. (v. 8-14)

Le poète s'amuse manifestement du contraste produit entre des expressions empruntées à la poésie de genre élevé, l'apostrophe dompna pour désigner la dame, la référence à sa pâmoison, et l'accumulation de termes grossiers et de situations triviales, les braies baissées, les draps bons à laver et l'activité sexuelle elle-même. Il faut cependant reconnaître que l'adoption du substantif «queue » et non de « bite », privilégié par Jean-Luc Leclanche dans sa traduction des fabliaux libertins ${ }^{17}$, était parfaitement subjective, si ce n'est que le terme « queue » présente l'avantage d'ajouter un jeu de mots ne détonnant pas avec la référence à l'âne en rut dans la même phrase. Le lexique de la traduction se veut ici trivial. Il rend viet par «queue », fotrai par «baiserai », coillon par « couilles », car le suffixe -on ne fonctionne

17. J. Dufournet préfère, comme nous, traduire vit par queue dans son édition bilingue des Fabliaux du Moyen Âge, Paris, 1998. Dans des traductions plus anciennes, les termes cons et vits disparaissent purement et simplement. Voir la traduction proposée par Jean Dufournet des v. 277-281 de Boivin de Provins, ibid.,p. 248 : «Pendant qu'elle cherchait, lui la tringlait ; il la piqua de la pointe de sa queue qu'il lui enfourna dans le con jusqu'aux couilles. Il lui battit et frappa le cul tant et tant, me semble-t-il, qu'il l'a bien baisée »; et la traduction élaborée par G. Rouger (Fabliaux, Paris, 1978, p. 130) : « et le vilain prend son plaisir ». 
manifestement pas comme un diminutif, au vu de l'adjectif gran qualifiant ces couilles, mais il possède plutôt une valeur affective. Le choix de ces termes renforce de manière plaisante l'écart instauré avec la forme poétique de la strophe que nous avons tenté de reproduire par des jeux d'échos sonores à la rime, des anaphores et des alexandrins au premier et au dernier vers ${ }^{18}$.

La traduction de vit par «queue » est encore celle choisie dans le fabliau de la Demoiselle qui ne pouvait entendre parler de foutre:

Lors li reprist a demander et ses choses a detaster, tant qu'el l'a par lo vit saisi et demande : «Que est ici, Davïet, si roide et si dur que bien devroit percier un mur ? » (v. 167-172)
Alors, elle commença à son tour à le questionner

et à tâter ses parties

tant et si bien qu'elle le saisit par la queue :

«Qu'est-ce, ici, David, demande-t-elle,

si raide et si dur

que cela pourrait bien percer un mur?»

L'auteur s'amuse à créer une dissonance entre le substantif choses, périphrastique et allusif, utilisé au moment où le tâtonnement de la pucelle est encore hésitant, et le substantif vit qui intervient quand elle se fixe enfin sur l'objet du delit. L'opposition de niveau de langue entre les substantifs «partie » et « queue » vise à rendre ce contraste qui contribue à la grivoiserie du passage.

Dans d'autres textes cependant, une traduction par « sexe » ou « verge », moins connotée, a reçu la préférence. Le fabliau du Souhait desvez n'est ainsi pas obscène. Il ne cherche pas à exposer de façon systématique ce que la bienséance interdit de montrer, à associer la chair aux plaisanteries scatologiques ou ordurières. Il prend pour protagonistes un couple tendrement épris et remplace la ruse, moteur traditionnel de ces contes, par le motif du songe traité de manière prosaïque. Sexuellement frustrée quand son époux sombre dans le sommeil après une absence de trois mois, la dame est assaillie en rêve par ses désirs et croit se promener sur un marché où sont vendus des sexes masculins plus énormes les uns que les autres :

Ainz n'i ot estal ne bojon ne n'i ot loge ne maison, changes ne table ne repair o l'an vandist ne gris ne vair, toile de lin ne drax de laine ne alun ne bresil ne graine ne autre avoir, ce li ert vis, fors solement coilles et viz. (v. 75-82)
Il n'y avait ni comptoir ni aune ni baraque ni magasin ni banc de changeur ni table ni éventaire où l'on vendît fourrures grises ou bigarrées, toile de lin, tissus de laine, mordant, bois de brésil, ou cochenille, ni autre denrée, lui semblait-il, à l'exception de couilles et de verges.

18. On peut ainsi comparer avec l'effet produit quand la strophe est traduite en prose par P. Bec (Burlesque..., p. 162) ou par P. Uhl («La tenso entre Montan et une Dame... »,p. 73) : « Et moi, Dame, je viens à vous la culotte baissée, avec un vit plus gros que celui d'un âne en rut, et je vous foutrai avec tant d'ardeur que vous devrez tordre les draps le lendemain. Vous constaterez alors qu'ils ont bien besoin d'aller à la lessive. Et jamais nous ne partirons, ni moi ni mes grosses couilles, tant que vous n'aurez pas été foutue au point d'en rester gisante et pâmée. » 
La substitution de sexes masculins aux marchandises habituellement proposées sur les comptoirs suffit à générer un effet comique souligné par le rejet des termes coilles et viz à la fin de l'énumération. Dans ce contexte, une traduction par « verge » a paru suffisante, même si elle échoue à rendre les jeux de mots créés en ancien français entre le pluriel viz et son homonyme, le substantif médiéval vis, " avis ». Elle se condamne également à ne pouvoir rendre la polysémie du titre, car ce Souhait desvez, insensé par ses outrances, est aussi un Souhait des vez, un rêve de verges, mais aucun terme contemporain ne permet de restituer ce double sens grivois ${ }^{19}$.

Dans le roman de Trubert, la traduction du terme vit dépend des phases de l'intrigue elles-mêmes. Quand le héros, sous une identité féminine, partage la couche de la fille du duc et l'initie au plaisir, traduire par « verge » ou « sexe » nous a semblé respecter le trouble s'emparant des personnages :

Et Trubert gist entre ses braz;

n'en puet mes se le vit li tent. (v. 2482-83)

Belement le tient et manoie,

et li viz en sa main coloie. (v. 2499-2500)

Sa main a mise contreval,

le vit a sesi par la teste. (v. 2548-49)
Et Trubert est allongé entre ses bras ;

il n'y peut rien si son sexe se dresse.

Elle le tient et le manie fort bien,

et la verge tend le cou sous sa main.

Elle descend la main

et saisit la verge par la tête.

La scène ne s'apparente pas à un coït rapide, mais elle décrit les douces caresses préliminaires prodiguées par la main de la demoiselle, elle évoque la découverte d'une intimité charnelle destinée à s'approfondir tendrement nuit après nuit. Alors que la sexualité de Trubert est à l'accoutumée dominée par des pulsions agressives, il renonce à toute brutalité en présence de cette jeune fille à la sensualité innée qui se montre aussi gourmande et insatiable que lui. Il en va autrement lors de la parodie de nuit de noces que subit Trubert avec le roi Golias. Le scandale qu'incarnent ces deux hommes réunis dans le même lit et l'obscénité de la scène justifient alors dans la traduction le recours au terme grossier « queue ».

De même, les deux emplois du substantif vit dans la Demoiselle qui songeait, ont généré deux traductions différentes, l'une neutre par « sexe », l'autre argotique par «queue », en fonction de leur insertion dans le récit ou dans le discours :

Tant quist que il trova lo lit.

Gros avoit et carré lo vit, et molt ert cointes lo ribaut. (v. 9-11)

«Cuidiez vos por vostre grant vit avoir moi si estoutoiee ?» (v. 58-59)

\author{
Il chercha tant qu'il trouva le lit. \\ Son sexe était gros et fort \\ et le vaurien était habile. \\ «Pensiez-vous avec votre grosse queue \\ m'avoir trop malmenée ?»
}

19. L. Rossi (Fabliaux érotiques..., p. 137-153) traduit ce fabliau en conservant le terme vit dans le titre et dans le texte, tout comme R. Brusegan (Fabliaux..., p. 104-115). G. Rouger (Fabliaux..., p. 137-140) traduit le titre en « Le Souhait contrarié », mais conserve le substantif vit dans toute la traduction. 
Lorsque la demoiselle fait allusion à la taille des attributs de son partenaire, son intention est clairement provocatrice : elle cherche à attiser son excitation afin de profiter à son tour de la situation, car il l'a auparavant violée quatre fois sans parvenir à la tirer du sommeil. Dans le récit en revanche, il nous semble que l'emploi du terme est moins connoté en raison de la présence au vers suivant de l'adjectif cointes, terme laudatif qui prête au protagoniste diverses qualités potentielles, l'habileté, la sagesse, la prudence, la bravoure, l'élégance ou la beauté ${ }^{20}$, auxquelles viennent s'ajouter comme naturellement des attributs de taille appréciable.

Le fabliau du Sot chevalier fait intervenir le terme vit à l'intérieur du discours que la belle-mère adresse au protagoniste afin de lui expliquer l'usage qu'il doit faire de son membre. On imagine mal cette vénérable dame, dont la narration signale par ailleurs la sagesse (v. 308), user d'un terme populaire ou familier en présence de ce gendre si empoté et le mot « verge » paraît conforme au niveau de langue qui pourrait être le sien. Il crée par ailleurs un contraste avec la traduction d'un autre substantif utilisé par l'auteur pour désigner le sexe de son personnage, la pasnaise (v. 48), le « panais », racine comestible dont l'emploi figuré et argotique perdure en français moderne ${ }^{21}$.

Traduire les termes référant aux parties basses du corps nécessite donc une constante attention à leur contexte afin de déterminer si, oui ou non, ils sont chargés de connotations populaires, scabreuses, grivoises ou obscènes, pour tenter de respecter au mieux la tonalité des extraits. Un problème similaire est posé par le verbe foutre. Il n'a rien de transgressif dans le Speculum al foderi, traité de médecine donnant à son lecteur des conseils afin de vivre une sexualité saine et propice à la génération. Dans ce cas d'ailleurs, nous aurions volontiers traduit le titre par le «Miroir du coït», si le texte n'était déjà connu et diffusé sous un intitulé plus aguicheur ${ }^{22}$.

Il semble en effet délicat de modifier les habitudes prises par les lecteurs ou les spécialistes pour désigner tel ou tel texte. La traduction du titre du fabliau de la Demoiselle qui ne pouvait entendre parler de foutre ne nous convient pas parfaitement et nous aurions préféré employer ici le substantif «baise ». Le public contemporain ne reconnaît sans doute pas l'infinitif substantivé désignant la relation sexuelle, mais il entend plutôt, à notre avis, le substantif renvoyant au sperme. Le maintien du verbe médiéval dans l'intitulé génère donc une ambiguïté nuisant à la compréhension

20. L. Rossi le traduit par « fougueux » dans Fabliaux..., p. 83.

21. On en trouve encore un exemple dans Mort à crédit de Céline (Paris, 1936, p. 326) : « Je la vois dans le blanc du réverbère !... En chemise de nuit... Toute redressée !... Ses cheveux qui flottent... Je reste là, moi, en berloque avec mon panais tendu. »

22. P. Gifreu a même cherché à renforcer cet effet en faisant précéder le Miroir du foutre par Le Kamasutra catalan dans la réédition de 2003. Celle d'octobre 2017 redonne au Miroir du foutre la première place. 
instantanée du sens que l'auteur a voulu donner à son conte, mais comme l'infinitif et le substantif appartiennent tous deux au même champ lexical et au même niveau de langue trivial, on peut estimer que la nuance est mince et ne nécessite pas de rebaptiser le fabliau.

Au cœur du texte lui-même en revanche et à l'exception du Speculum al foderi où les emplois du verbe foder relèvent du traité de sexologie, nous avons choisi de traduire foutre par « baiser », et non « tringler », « entuber », « ramoner » ou «troncher », par exemple, car «baiser» présente l'avantage de posséder parmi ses paradigmes morphologiques le substantif «baise », idéal pour traduire l'infinitif foutre quand il est substantivé ${ }^{23}$. Une autre qualité du verbe baiser est sa polysémie qui convient à merveille pour traduire le titre du fabliau de la Demoiselle qui songeait qu'on la baisait, car le français moderne baiser superpose le sens neutre d' « embrasser » et le sens grivois. Cette syllepse de sens n'existe pas dans le titre médiéval nettement plus explicite, De la Damoisele qui sonjoit qu'en la fotoit, mais elle correspond en tous points au rêve initial de l'héroïne qui songe qu'un biax bacheliers épris d'elle vient la retrouver dans son lit, alors que le personnage masculin s'avère être un pautonier, un « vaurien », qui profite de l'obscurité pour se faufiler dans sa demeure, dans son lit et plus loin encore. L'emploi du verbe baiser dans le titre français correspond ainsi à la fois au rêve d'amour éthéré de l'héroïne et à la réalité scabreuse qui lui succède.

Un cas de conscience différent se pose avec la traduction du substantif lecherie. Quand il apparaît dans Le Bel Inconnu, roman arthurien du XIII ${ }^{\mathrm{e}}$ siècle, et qu'il est placé dans le discours de la fée de l'Île d'Or qui a rejoint le héros éponyme sur sa couche mais refuse finalement de se donner à lui, il est facile d'opter pour une traduction de style soutenu comme «débauche », «stupre » ou « dépravation » ${ }^{24}$, conforme aux sens mentionnés par le dictionnaire de Godefroy ${ }^{25}$. Mais dans des textes où le terme fonctionne manifestement selon un registre de langue moins soutenu, comme la Demoiselle qui ne pouvait entendre parler de foutre ou Richeut, il a semblé préférable de le traduire par « coucherie », mot présentant l'avantage de comporter des sonorités voisines. Il a fallu néanmoins accepter que se

23. Les usages des traducteurs sont divers. Certains suppriment les passages où le verbe foutre apparaît, comme A. Jeanroy et G. Rouger. D'autres maintiennent le verbe foutre dans la traduction, comme R. Brusegan, L. Rossi, P. Uhl. P. Bec alterne le verbe foutre et le verbe baiser. J. Dufournet est le seul à le traduire dans Boivin de Provins par «baiser » et « tringler », traduction justifiée par la brutalité de l'acte dans ce fabliau (Fabliaux..., p. 248-249). On notera que la tendance générale de L. Rossi est de conserver le plus possible les termes médiévaux dans la traduction en français moderne, puisqu'il garde également le verbe baiser même quand il a le sens de «embrasser » (voir par exemple Fabliaux..., p. 74-75, v. 46).

24. M. Perret et I. Weill traduisent le v. 2451 : Tot torneroit a lecerie ! par « Quelle indécence ! », dans Renaud de Beaujeu. Le Bel Inconnu, Paris, 2003, p. 147.

25. F. Godefroy, Dictionnaire..., vol. IV, p. 751, [en ligne] : http://micmap.org/dicfro/ search/dictionnaire-godefroy/lecherie [consulté le 19 septembre 2018]. 
perdent les références à la gourmandise, à la gloutonnerie alimentaires et sexuelles contenues dans le substantif médiéval ${ }^{26}$. Le terme coucherie permet toutefois de créer des jeux sonores avec d'autres substantifs grivois, aptes à concurrencer les rimes du texte médiéval :

... en nul sen ne sofrist mie sergent qui nomast lecherie, vit ne coille ne autre chose.

(Demoiselle qui ne pouvait entendre parler de foutre, v. 21-23)
... elle n'aurait jamais supporté un serviteur qui eût parlé de coucherie, de queue, de couille ou d'autre chose.

L'énumération des substantifs coucherie, queue et couille produit dans la traduction une allitération qui tente de suppléer à l'absence d'un même rythme versifié et des mêmes sonorités.

Un cas particulier est présenté par l'emploi du terme lecherie au vers 103 du fabliau de la Saineresse, car il y est utilisé en syllepse pour référer à la fois au moment de débauche que s'est octroyé la bourgeoise avec un amant déguisé en femme médecin et au bon tour qu'elle joue à son mari en lui contant par le menu ses ébats sous le couvert de la métaphore médicale. Godefroy signale que le substantif lecherie possède parfois le sens de «bon tour $\gg^{27}$. Nous aurions aimé trouver un substantif en français moderne qui aurait connoté dans le même temps la ruse et la débauche, mais en l'absence d'un tel mot, il a fallu recourir à une périphrase et remplacer essaucier la lecherie par "glorifier comment elle l'avait trompé », ce verbe étant assez polysémique pour évoquer simultanément le fait d'abuser autrui et de lui être infidèle. C'est aussi lui qui traduit l'infinitif bouler utilisé dans le même fabliau ${ }^{28}$. Godefroy donne seulement pource verbe le sens de «tromper», mais il signale que le substantif bolerie peut désigner la débauche ${ }^{29}$. Un verbe d'un niveau de langue moins soutenu que « tromper » eût été préférable, mais en français moderne, les infinitifs argotiques comme «baiser », « couillonner » ou « entuber » associent la duperie au fait d'être sexuellement possédé, non au fait de tromper son conjoint. En utilisant l'infinitif bouler, l'auteur use de surcroît d'un jeu de mots intraduisible avec un homonyme qui avait le sens de « rouler comme une boule ${ }^{30} »$, mais traduire bouler par « rouler » aurait eu le tort de masquer toutes les connotations sexuelles.

26. C'est également la traduction proposée par L. Rossi (Fabliaux..., p. 93, v. 7 et 22, et p. 97 v. 94). R. Brusegan préfère traduire lecerie par « paillardise» (Fabliaux..., p. 203) et par « propos grivois » (ibid., p. 207).

27. Et c'est ainsi que traduit L. Rossi (Fabliaux..., p. 79).

28. V. 3, 109 et 116. Comme nous, L. Rossi traduit le verbe bouler par « tromper» (Fabliaux..., p. 73 et 79).

29. F. Godefroy, Dictionnaire..., vol. I, p. 678 [en ligne] : http://micmap.org/dicfro/ search/dictionnaire-godefroy/boler [consulté le 19 septembre 2018].

30. Ibid., p. 701, [en ligne] : http://micmap.org/dicfro/search/dictionnaire-godefroy/ bouler [consulté le 19 septembre 2018]. 
L'emploi du substantif lecherie dans le fabliau de la Saineresse génère un autre jeu sémantique impossible à restituer en français moderne. La bourgeoise, qui se plaint de souffrir d'une effroyable crise de goutte aux reins, ne se contente pas de narrer à son mari comment elle a été soignée par l'application de prétendues ventouses pour la saigner. Elle ajoute encore que ses plaies ont été enduites d'un merveilleux onguent dont elle précise la provenance :

« Et si ne vous en quier mentir, l'oingnement issoit d'un tuiel et si descendoit d'un forel d'une pel molt noire et hideuse, mes molt par estoit savoreuse. » (v. 92-96)

\author{
«Et sans vouloir vous mentir, \\ l'onguent sortait d'un tuyau \\ et descendait d'un fourreau \\ en peau noire et hideuse, \\ mais extrêmement savoureuse. »
}

Avant de se doter d'un sens figuré, l'adjectif savoureuse implique un goût agréable en bouche et il paraît difficile de ne pas voir en lui une allusion à des pratiques sexuelles orales qui justifient un peu plus encore l'utilisation dans le fabliau du terme lecherie, dont le double sens se perd hélas dans la traduction.

Restait à savoir comment traduire le substantif de la même famille, le leceor. Il était à l'évidence exclu d'utiliser le terme « coucheur » qui n'a pas de sens érotique en français moderne. On aurait pu proposer nombre de traductions d'un niveau de langue élevé, « débauché31, " « dépravé », « libertin », mais à force d'hésiter entre " noceur », qui ne réfère pas exclusivement aux appétits sensuels, et « coureur », trop ambigu, nous avons opté, dans Richeut, pour «baiseur » et, dans la Demoiselle qui ne pouvait entendre parler de foutre, pour « cochon », qui n'est pas si éloigné phonétiquement de « coucherie » et dont le sens moderne de «personne portée au vice, à la débauche, manquant de décence dans ses actes, ses propos, ses écrits ${ }^{32} »$ nous paraît parfait dans le contexte.

On le voit, en dépit de nos efforts pour restituer la signification précise des termes utilisés par nos auteurs, il se produit toujours des déperditions de sens qui sont encore plus importantes quand les textes jouent sur les différentes valeurs d'un même mot en syllepse ou qu'ils réfèrent à des réalités aujourd'hui disparues. Le substantif saineresse désignant une femme médecin chargée d'effectuer les saignées ne connaît pas d'équivalent à l'heure actuelle et traduire le titre du fabliau oblige à user d'une périphrase qui masque l'ambiguiité présente dans la langue médiévale entre le verbe sainier, « saigner », et son homonyme sainer, « soigner »,

31. C'est la traduction adoptée par L. Rossi (Fabliaux..., v. 81, p. 97). R. Brusegan (Fabliaux..., p. 207) choisit de traduire qu'i sont lecheor par « qui soient dégourdis ».

32. Voir le Trésor de la langue française, [en ligne] : http://www.cnrtl.fr/definition/ cochon [consulté le 19 septembre 2018]. 
pris dans un sens érotique ${ }^{33}$. La Confession de Margot est une farce à deux personnages de la fin du $\mathrm{Xv}^{\mathrm{e}}$ siècle parodiant sur un mode grivois le rite chrétien de la confession. Margot se repent de ses multiples péchés et implore le pardon divin, tandis que son confesseur s'entête à ne voir dans son attitude luxurieuse et lascive qu'un effet de sa générosité d'âme, sauf dans un seul passage, quand la jeune femme décrit sa réaction en présence de la belle chose qu'un ermite tenait entre ses doigts :
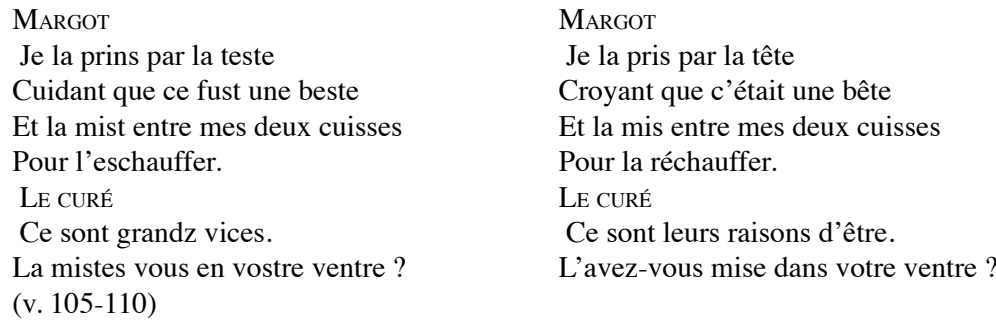

On pourrait croire ici que le substantif vice est pris dans son sens moderne de « disposition au mal », mais en fait, il existait dans l'ancienne langue un homonyme féminin signifiant "fonction, raison d'être ». L'adjectif grandz ne permet pas de déterminer le genre du substantif, car il était épicène et n'a été doté d'un $e$ au féminin qu'à partir du moyen français. Le traducteur est donc condamné à opter pour l'un ou l'autre de ses sens qui sont antinomiques, alors que la farce joue sur la syllepse ${ }^{34}$. Comme le curé ne condamne jamais par ailleurs l'attitude de Margot et qu'il n'y décèle que des actions recommandables, nous avons traduit, tout en regrettant une fois encore que la traduction soit condamnée à simplifier la subtilité de la langue médiévale qui pimentait l'agrément de l'auditoire d'une note piquante de plaisir intellectuel.

Il eût été plus simple de ne pas traduire les textes érotiques du Moyen Âge et d'abandonner le lecteur à ses erreurs d'interprétations, ses hésitations et ses questionnements. Mais cette anthologie a été pensée pour répondre à la nécessité de faire connaître ces extraits et de montrer la modernité dont ils témoignent dans la représentation de la sexualité. Traduire s'est alors imposé comme une obligation, même si cette démarche a généré aussitôt bon nombre d'interrogations nouvelles. Quels termes existant en ancien français fallait-il conserver ? Quels étaient les mots exigeant être traduits? Et dans ce cas, la traduction devait-elle privilégier

33. Voir F. GoderRoy, Dictionnaire ..., vol. VII, p. 304, [en ligne] : http://micmap.org/ dicfro/search/dictionnaire-godefroy/saner [consulté le 19 septembre 2018].

34. A. Tissier, qui édite cette farce dans le tome VI de son Recueil de farces (1450-1550), Genève, 1990, p. 369-422, ne signale pas la syllepse en note. 
un niveau de langue familier ou vulgaire, grivois ou obscène ? Fallait-il maintenir le verbe foutre dont le sens s'est modifié ou le substantif vit qui n'est majoritairement plus compris, quitte à obliger nos contemporains à tenir l'anthologie érotique d'une main et le dictionnaire de l'autre ? Il est alors certain que l'effet recherché par les auteurs médiévaux se serait perdu, que la part de transgression liée à l'évocation crue de la sexualité se serait délitée devant les obstacles posés par l'élucidation du sens. Traduire l'ancien français est devenu une nécessité si l'on veut permettre au lecteur moderne de partager les réactions de l'auditoire médiéval face à ces textes provocateurs. Cette entreprise présente cependant l'avantage de dévoiler les mécanismes complexes se jouant autour de la transposition littéraire de l'activité sexuelle, car en définitive, l'obscénité, la parodie, les doublesens grivois sollicitent l'intelligence et la vivacité d'esprit de l'auditoire, du lecteur et du traducteur tout autant que leur imaginaire érotique. Le jeu des corps ainsi exposé se double d'un jeu intellectuel et fantasmatique qui signe à nos yeux ce qu'est véritablement l'érotisme.

\section{Corinne Pierreville - Université Jean Moulin-Lyon 3, UMR 5648 (CIHAM)}

\section{Le choix des mots. Traduire les textes érotiques du Moyen Âge}

La traduction est, comme chacun sait, un exercice condamnant le traducteur à une forme de frustration : il est impossible de restituer parfaitement toutes les nuances d'un texte, les connotations d'un mot, le rythme, les sonorités, les suggestions phoniques et sémantiques, le style d'un auteur. Les difficultés de cette entreprise s'accroissent quand le texte source relève d'une littérature s'attachant à l'évocation du désir et du plaisir sexuel. Lors de la constitution d'une anthologie de la littérature érotique médiévale, la nécessité de la traduction s'est imposée afin d'éviter de surcharger les textes de notes sémantiques, tant la langue du Moyen Âge est devenue étrangère au lecteur contemporain. Traduire était nécessaire pour éviter au lecteur des erreurs de compréhension et lui donner les clés du sens. Mais comment traduire ? Quels termes existant en français moderne fallait-il conserver ? Quels étaient les mots exigeant d'être traduits ? Des exemples précis nous permettent de justifier les choix qui ont été les nôtres tout en évoquant les résistances que nous avons rencontrées et, en particulier, les jeux de syllepse et de double-sens que nous avons échoué à traduire.

comique, érotisme, obscénité, traduction, transgression

\section{A choice of words ? Translating the erotic texts of the Middle Ages}

As everyone knows, translation is an exercise that can lead the translator to become frustrated, since it is not possible to perfectly restore all the various nuances of a text, the connotations of a word, the rhythm, the tones, the phonic and semantic suggestions, and the style of an author. These difficulties increase when the writing deals with sexual desire and pleasure. In order to provide an anthology of medieval erotic literature, translation is essential, since the contemporary reader would be unable to understand the language of the Middle Ages, and the original text would have to be heavily 
annotated with notes on meaning. Translation is necessary for enabling the reader's understanding of the text, and for providing keys to its meaning. But how should we translate? What terms that exist in modern French should we keep ? What words should we change ? In this article, we use specific examples to justify our choices, as well as discussing what we failed to translate.

comedy, eroticism, obscenity, transgression, translation 